\title{
Evaluation of the rheological parameters modification of concrete vibrating compaction by dynamic methods
}

\author{
P. Bratu ${ }^{1}$ and R. Pintoi ${ }^{1}$ \\ ${ }^{1}$ ICECON SA, Research Institute for Construction Equipment and Technology, Bucharest, Romania
}

\begin{abstract}
The paper presents the rheological modelling of fresh concrete subjected to vibrations during the compacting process. The compacting efficiency can be estimated by means of the vibration transmissibility from the vibrating tool to the fresh concrete mass. Also the experimental results are presented.
\end{abstract}

\section{Introduction}

The behaviour of fresh concrete is a fundamental technological element in the process of assuring the quality performances concerning both the strength and the porosity of the concrete structures.

Optimal vibration parameters are required for the vibration regime in order to reach a certain degree of compactability of the concrete.

In order to obtain a vibration regime optimally adapted to particular concrete receipt, the analyst must use multicriterial correlation specifically adapted to each studied receipt.

This study tries to avoid some of the traditional procedure disadvantages by introducing a global parameter to evaluate the vibrations effect upon the degree of compactability of the concrete. Thus we introduce the synthetic parameter vibration transmissibility $\mathrm{T}$ from the vibrating body to the fresh concrete subjected to vibration. The fresh concrete is considered to be a rigid body connected to the vibrating body by an exclusively linear viscous link whose elasticity factor can be neglected.

\section{Analysis of the compacting degree in the case of fresh concrete subjected to the action of forced harmonic vibrations}

The analyses of the system actuated by a periodic force acting on a single direction will take into account the concrete movement which takes place with a phase difference related to the frame that is fixed onto the equipment. The mathematical model is presented in figure 1.

The differential equations of the motion are:

$\left\{\begin{array}{l}m_{1} \ddot{x}_{1}+k x_{1}+c\left(\dot{x}_{1}-\dot{x}_{2}\right)=F_{o} \cos \omega t \\ m_{2} \ddot{x}_{2}-c\left(\dot{x}_{1}-\dot{x}_{2}\right)=0\end{array}\right.$

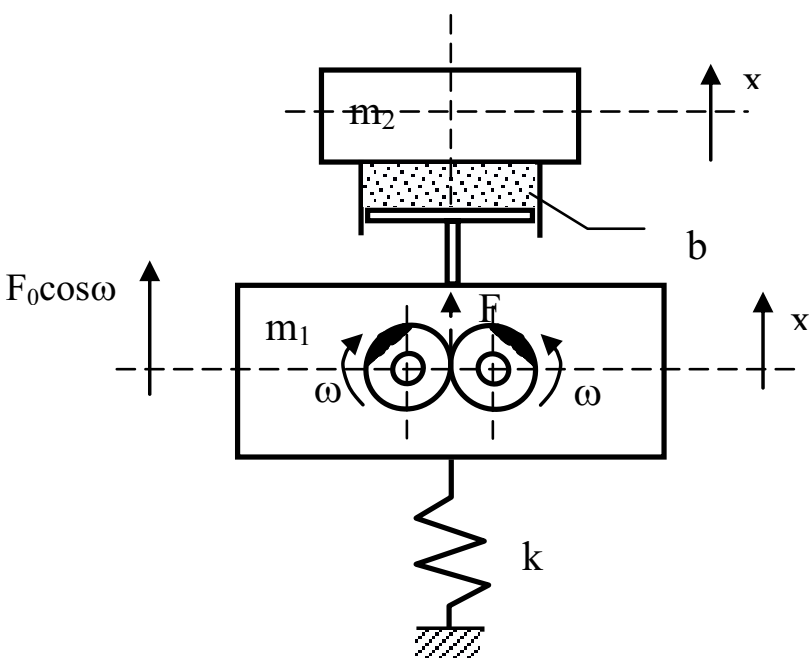

Fig1: The calculus dynamic model

The complex amplitudes $\widetilde{A}_{1}$ and $\widetilde{A}_{2}$ will have the expressions:

$$
\begin{aligned}
& \widetilde{A}_{1}=\frac{\widetilde{F}}{D^{2}+E^{2}}[(G D+E H)+j(D H-E G)] ; \\
& \widetilde{A}_{2}=-\frac{\widetilde{F}}{D^{2}+E^{2}}(E+j D)
\end{aligned}
$$

Where:

$G=-m_{2} \omega^{2}$ and $H=c \omega . \quad \mathrm{D}, \mathrm{E}, \omega, \mathrm{F}_{0}$

The amplitudes $\mathrm{A}_{1}$ and $\mathrm{A}_{2}$ of the complex $\widetilde{A}_{1}$ and $\widetilde{A}_{2}$ are obtained as: 
$A_{1}=\frac{F_{o}}{\Delta} \sqrt{G^{2}+H^{2}} ; A_{2}=\frac{F_{o} H}{\Delta}$

By replacing D, E, G, H, $F_{o}=m_{o} r \omega^{2}$ the expressions of $\Delta, \mathrm{A}_{1}$ and $\mathrm{A}_{2}$ can be written:

$\Delta=\omega R$

Where:

$R=\sqrt{r_{6} \omega^{6}+r_{4} \omega^{4}+r_{2} \omega^{2}+r_{o}}$

The factors $r_{n}, n=0,2,4,6$ are given by the relations:

$r_{o}=k^{2} c^{2}$

$r_{2}=k^{2} m_{2}^{2}-2 k c^{2}\left(m_{1}+m_{2}\right) ;$

$r_{4}=c^{2}\left(m_{1}+m_{2}\right)^{2}-2 m_{1} m_{2}^{2} k ; r_{6}=m_{1}^{2} m_{2}^{2}$

$A_{1}=\frac{m_{o} r \omega^{2}}{R} \sqrt{m_{2}^{2} \omega^{2}+c^{2}} ; A_{2}=\frac{m_{o} r \omega^{2}}{R} c$

In the case of a working regime stabilised at a working frequency over the resonance which means $\omega>>p_{\max }$ and $\omega \rightarrow \infty$ it results in:

$A_{1}^{\text {stab }}=\lim _{\omega \rightarrow \infty} A_{1}=\frac{m_{o} r}{m_{1}} ; A_{2}^{\text {stab }}=\lim _{\omega \rightarrow \infty} A_{2}=0$

The compacting degree of the concrete can be appreciated by the force transmissibility which is a synthetic parameter defined as follows:

$$
T=\frac{F_{T}^{\max }}{F_{0}}
$$

Where

$F_{T}^{\max }$ is the maximum force transmitted to the concrete; $F_{o}=m_{o} r \omega^{2}$ is the maximum disturbing force.

By choosing the real solutions of the system to have the form:

$x_{1}=A_{1} \cos \omega t ; x_{2}=A_{2} \cos (\omega t-\varphi)$

Where

$\varphi$ is the angle between the disturbing force $F(t)=F_{o} \cos \omega t$ and the instant displacement $\mathrm{x}_{2}$ and is given by the relation $\operatorname{tg} \varphi=\frac{m_{2} \omega}{c}$.

The transmitted force will be:

$F_{T}=c\left(\dot{x}_{1}-\dot{x}_{2}\right)=c \omega\left[\left(A_{1}+A_{2} \cos \varphi\right) \sin \omega t-A_{2} \sin \varphi \cos \omega t\right]=$

$F_{T}^{\max } \sin (\omega t+\theta)$

and it results in:
$F_{T}^{\max }=c \omega \sqrt{A_{1}^{2}+A_{2}^{2}+2 A_{1} A_{2} \cos \varphi} \quad$ and

$\operatorname{tg} \theta=-\frac{A_{2} \sin \varphi}{A_{1}+A_{2} \cos \varphi}$

The force transmissibility to concrete mass will finally be expressed in the form:

$T=c \sqrt{\frac{m_{2}^{2} \omega^{4}+4 b^{2} \omega^{2}}{r_{6} \omega^{6}+r_{4} \omega^{4}+r_{2} \omega^{2}+r_{o}}}$

The transmissibility expression shows that it essentially depends upon the factor $\mathrm{b}$. The function $\mathrm{T}=\mathrm{T}(\omega)$ has a maximum for the single real $\omega$ solution of the denominator. There are two other imaginary solutions.

Figure 5 presents the variation of transmissibility $\mathrm{T}$ versus $\omega$ for two dynamic regimes defined by $\Omega=\frac{\omega}{p}$, so that a stable regime be possible at distinct values of transmissibility. For discreet values of exciting pulsation we have the values of parameter $\Omega$.

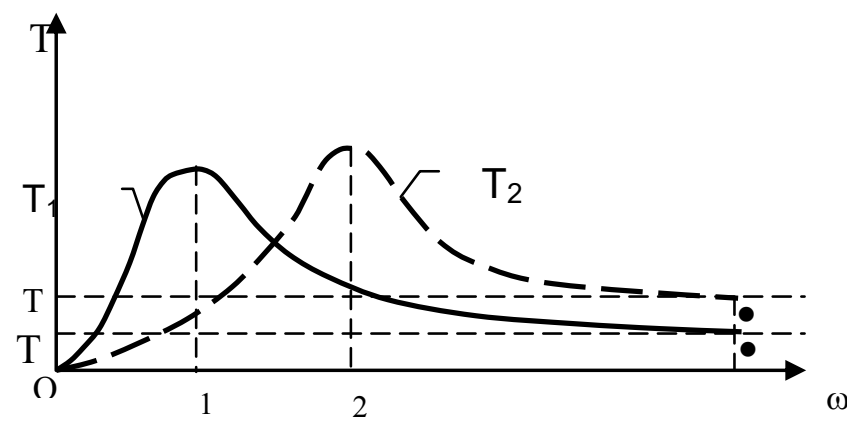

Fig 2: The variation of transmissibility

In figure 2 are illustrated two resonance diagrams $T_{1}$ and $T_{2}$ for eigen value $p_{1}$ and $p_{2}$ which correspond to two different samples of same concrete, with different masses $\mathrm{m}_{1}$ and $\mathrm{m}_{2}$. For the sample $\mathrm{m}_{1}>\mathrm{m}_{2}$, force transmitted to compactability with pulsation $\omega$, we have transmissibility $T_{1}<T_{2}$. The compactability degree for the sample with bigger mass will be realised after a longer vibration period.

\section{Energy dissipated during the vibrating compacting process}

The elementary mechanical work performed by the generalised force $Q_{\varphi}$ corresponding to the actuation moment of the vibro-generator for an elementary angle $\mathrm{d} \varphi$ is defined by relation:

$\mathrm{d} L=Q_{\varphi} \mathrm{d} \varphi$

Where 
$Q_{\varphi}=\frac{1}{2} m_{0} r A_{1} \omega^{2}\left[\sin \theta_{1}+\sin \left(2 \omega t-\theta_{1}\right)+m_{0} g r \sin \omega t\right]$

Where

$$
\varphi=\omega t \text { and } d \varphi=\omega d t
$$

so

$$
\Delta w_{d}=L=\int_{0}^{2 \pi} Q_{\varphi} d \varphi
$$

We will obtain

$$
\Delta w_{d}=\pi c \omega A_{1}^{2}
$$

Where

$$
\begin{aligned}
& A_{1}=A_{1}^{s t a b}=\frac{m_{1} r}{m_{1}} \\
& c=2 \zeta m_{1} p \\
& \zeta=\frac{c}{c_{r}}
\end{aligned}
$$

Dissipated energy relation is

$$
\begin{aligned}
\Delta w_{d} & =\pi c \omega\left(\frac{m_{0} r}{m_{1}}\right)^{2} \\
\Delta w_{d} & =2 \pi p \zeta \omega \frac{\left(m_{0} r\right)^{2}}{m_{1}}
\end{aligned}
$$

The loss factor is:

$\eta=\frac{\Delta w_{d}}{2 \pi w_{e l}^{\max }}$

Where

$$
w_{e l}^{\max }=\frac{1}{2} k A^{2}=\frac{1}{2} m_{1} p^{2}\left(\frac{m_{0} r}{m_{1}}\right)^{2}=\frac{1}{2} p^{2} \frac{\left(m_{0} r\right)^{2}}{m_{1}}
$$

and finnaly we have

$$
\eta^{r e z}=\frac{\Delta w \cdot m_{1}}{\pi p^{2}\left(m_{0} r\right)^{2}}
$$

At resonance $\omega=\mathrm{p}$, so $\eta^{\mathrm{rez}}=2 \zeta_{\mathrm{eq}}$ experimental values were determined stiffness and damping characteristics presented in table 1 .

c) In forced vibration duty with controlled force, with known values for exciting pulsation $\omega$ and static moment $\mathrm{m}_{0} \mathrm{r}$ were determined the amplitude $\mathrm{A}_{1}$ and pulsation $\omega=(3 \ldots 4) p$.

d) According to measured values and calculated values $\mathrm{m}_{1}$ concrete mass and the static moment $\mathrm{m}_{0} \mathrm{r}$, was established the dissipated energy by calculation and by experimental evaluation. In this case the dissipated energy in constant condition increases with increasing of exciting pulsation and decreasing of vibrated concrete.

\section{References}

1. P. Bratu, "Vibratii mecanice", 30-86 (Editura Impuls, 1998)

2. C. Hu, G.I. Pelova and J.C. Walraven, "Characterizing the properties of fresh concrete.BML-viscosimeter and BTRHEOM rheometer comparative experiments",274-275 (Proceedings of the Fifth European Rheology Conference, University of Ljubljana, Slovenia, 1998)

3. K.D. Wantke and H. Fruhner, "The relationship between foam stability and surface rheological properties", 315-316 (Proceedings of the Fifth European Rheology Conference, University of Ljubljana, Slovenia, 1998)

4. SR EN 206-1:2002, "Beton. Partea 1: Specificatie, performanta, productie si conformitate"

5. SR EN 12350-4:2002, "Incercare pe beton proaspat. Partea 4: Grad de compactare".

\section{Conclusions}

According to the tests performed with fresh concrete with two receipts C 8/10 and C 20/25 were obtained:

a) For free vibration generated by an initial impulse enough to put in motion the entire vibrant mass with fresh concrete. Was registered damping vibro-diagram for free motion.

b) Was highlighted viscous character of concrete in compaction process. In this case, using specific 